Published by Al-Nahrain College of Medicine P-ISSN 1681-6579

E-ISSN 2224-4719

Email: iraqijms@colmed-alnahrain.edu.iq

http://www.colmed-alnahrain.edu.iq

http://www.iraqijms.net

Iraqi JMS 2019; Vol. 17(1)

\title{
Pediatric Hodgkin Lymphoma in Iraq-KRG-Sulaimani
}

\author{
Basil K. Abdallah ${ }^{1} M D$, Nawshirwan G. Rashid² $M D$, Shwan A. Tawfiq ${ }^{2} M D$ \\ ${ }^{1}$ Dept. of Pediatric Hematology and Oncology, Hiwa Hemato-Oncology Hospital, Iraq-KRG-Sulaimani, ${ }^{2}$ Dept. of Clinical \\ Hematology, Hiwa Hemato-Oncology Hospital, Iraq-KRG-Sulaimani
}

\begin{abstract}
Background Hodgkin lymphoma $(\mathrm{HL})$ is a highly curable malignancy. It is a unique neoplasm, in which the malignant cell is the Reed-Stenberg cell (RSC); it constitutes approximately $40 \%$ of all lymphomas that present during childhood.

Objective To obtain local data on the pattern of childhood and adolescent $\mathrm{HL}$ in our community at presentation and then compare it with the international figure.

Methods Eighty-five patients with newly diagnosed pediatric HL were admitted to Hiwa Hematology/Oncology Hospital in Sulaimaniya province of Iraqi Kurdistan were included in this study, they were studied prospectively from March 2006 to March 2014. The patients staged by Ann Arbor Staging system and categories into three risk group depending on the Stage and Number of Nodal Sites and the presence of Bulky Disease. Data analyzed using SPSS software; version 13 and P-value obtained by Chi-square test.

Results The median age at diagnosis was about 13.7 years with peak age of incidence was between 15-18 year and with male predominance. Most common site of the primary tumor was cervical lymph nodes; nodular sclerosis was the most common histopathological subtype and the majorities were presented in stage IIA.

Conclusion The stage in this study was age dependent but neither there was relation between the site of the primary tumor and the risk categories nor relation between risk categories and sex.

Keywords

Citation Hodgkin lymphoma, stage, sex distribution, risk category

Abdallah BK, Rashid NG, Tawfiq SA. Pediatric Hodgkin lymphoma in Iraq-KRG-Sulaimani. Iraqi JMS. 2019; 17(1): 12-17. doi: 10.22578/IJMS.17.1.3.
\end{abstract}

List of abbreviations: $\mathrm{HL}=$ Hodgkin Iymphoma, $\mathrm{LDHL}=$ Lymphocytic depletion Hodgkin lymphoma, LRCHL = Lymphocytic rich cellularity Hodgkin lymphoma, $\mathrm{MCHL}=$ Mixed cellularity Hodgkin lymphoma, NLPHL = Nodular lymphocytic predominant Hodgkin lymphoma, NSHL = Nodular sclerosis Hodgkin lymphoma

\section{Introduction}

$\mathrm{F}$ lormally known as Hodgkin's disease, Hodgkin lymphoma (HL) is a highly curable malignancy. It is a unique neoplasm in which the malignant cell, the ReedStenberg cell (RSC), represents only a small proportion of cells constituting the bulk of the tumor. It also has very particular clinical characteristics and distinct biological behavior. $\mathrm{HL}$ is a rather rare malignancy in the pediatric population; however, it constitutes approximately $40 \%$ of all lymphomas that present during childhood and is the most common malignancy in adolescents and young adults. In all age groups, $\mathrm{HL}$ is highly sensitive to chemotherapy and irradiation. In fact, HL was the first cancer to be cured with radiation therapy alone or with a combination of several chemotherapeutic agents. The cure rate for children and adolescents with $\mathrm{HL}$ has steadily improved over the years, particularly with the introduction of combined radiation and multiagent chemotherapy ${ }^{(1)}$. This therapeutic success has come at the price of serious long- 
term toxicities, such that a 30-year survivor of $\mathrm{HL}$ is more likely to die of therapy-related complications rather than from HL. Therefore, the therapeutic paradigm has shifted toward reducing treatment-associated toxicity while maintaining high cure rates. This new paradigm has led to the current risk-adapted, responsebased approach to the treatment of $\mathrm{HL}^{(2-4)}$.

This study aimed to obtain local data on the pattern of childhood and adolescent Hodgkin lymphoma in our community at presentation and then to compare it with the international figure.

\section{Methods}

Eighty-five patients with newly diagnosed pediatric and adolescent $\mathrm{HL}$ were admitted to Hiwa Hematology/Oncology Hospital in the Sulaimaniya province of Kurdistan/ Iraq. They were studied prospectively from March 2006 to March 2014.

\section{Inclusion criteria}

1. All children and adolescents aged 18 years or younger.

2. Randomly collected regardless to the gender.

3. Histopathologically proved HL.

4. Newly diagnosed patients who were not treated previously by chemotherapy.

\section{Exclusion criteria}

1. Age more than 18 years.

2. Relapsed HL or previously treated with chemotherapy.

\section{Study requirements}

All the targeted patients had their Sulaimaniya facilities of hitopathological diagnosis, complete blood count (CBC), blood film, erythrocyte sedimentation rate (ESR), liver function test, serum alkaline phosphates, hepatitis B serology, hepatitis $C$ serology, human immunodeficiency virus serology, renal function test, serum electrolyte, serum lactic dehydrogenase (LDH), serum ferritin level, abdominal ultrasound, chest X-ray, echocardiography, computerized tomography (CT)-scan and/or magnetic resonance imaging (MRI) of the primary site, chest and abdomen, bone marrow aspirate and trephine biopsy was done for those with one or more of the following criteria:

1. Patients with clinical stage III or stage IX.

2. Patients with B symptoms.

3. Patients with cytopenia on $\mathrm{CBC}$.

4. Patients with elevated serum alkaline phosphates.

Positron emission tomography (PET) scan and Epstein-Barr (EB) virus Study were not done for the majority of the case because it was not available.

The patients were staged according to the Modified Ann Arbor Staging system ${ }^{(1,5,6)}$.

Stage I

Involvement of a single lymph node region or lymphoid structure.

Stage II

Involvement of two or more lymph node regions or localized involvement of one extranodal site and one or lymph node regions, all on the same side of the diaphragm.

Stage III

Involvement of lymph node regions or structures on both sides of the diaphragm.

Stage IV

Diffuse or disseminated involvement of one or more extralymphatic organs, or isolated extralymphatic organ involvement without adjacent regional lymph node involvement, but with disease in distant site(s), or any involvement of the liver, bone marrow, pleura or cerebrospinal fluid (CSF).

According to their $\mathrm{HL}$ risk categories depending on the stage and number of nodal sites and the presence of bulky disease, favorable-risk pediatric HL (stage IA or IIA with $<3$ nodal sites, and some IIIA without bulky disease), intermediate-risk disease (stage IIA bulky disease with extension or $=3$ nodal sites, stage IB, IIB, stage IIIA, stage IVA) and advanced or unfavorable pediatric $\mathrm{HL}$ (all other patients that were not included in the favorable or the intermediate risk groups).

Data analyzed using Statistical package for social sciences (SPSS) software; version 13 and $P$-value obtained by Chi-square test, $P$ value less than 0.05 considered as significant. 


\section{Results}

Among 85 patients with HL studied, 59 patients (69.41\%) were males and 26 patients (30.58\%) were females. The male:female ratio was 2.27:1.

A median age at diagnosis was about 13.7 years with peak age of incidence was between 15-18 years. Table 1 shows the age distribution.

Table 1. Age distribution of Hodgkin's lymphoma at diagnosis

\begin{tabular}{ccc}
\hline Age in years & No. of patients & Percent (\%) \\
\hline $0-4$ & 4 & 4.7 \\
$5-9$ & 17 & 20.0 \\
$10-14$ & 25 & 29.4 \\
$15-18$ & 39 & 45.9 \\
\hline
\end{tabular}

The most common site of the primary tumor was the cervical lymph nodes, which were the primary site of the tumor in 48 out of 85 patients $(56.47 \%)$, followed by isolated mediastinal primary which occurred in 13 patients (15.29\%), axillary lymph nodes primary in 8 patients $(9.41 \%)$, primary inguinal lymph nodes involvement occurred in 7 patients (8.23\%), retroperitoneal lymph nodes primary in 5 patients $(5.88 \%)$ and isolated splenic involvement occurred in 4 patients (4.7\%).

Among the 85 patients with $\mathrm{HL}$ studied, 44 patients $(51.76 \%)$ had nodular sclerosis $\mathrm{HL}$ (NSHL), 23 patients (27.05\%) had mixed cellularity $\mathrm{HL}$ (MCHL), 12 patients (14.11\%) had lymphocytic rich cellularity $\mathrm{HL}$ (LRCHL), 1 patient (1.17\%) had Lymphocytic depletion HL (LDHL) and 5 patients (5.88\%) were nodular lymphocytic predominant HL (NLPHL).

Regarding modified Ann Arbor Staging of the studied patients, 9 patients (10.59\%) were in Stage I, three of them (3.53\%) with B symptoms, 37 patients (43.53\%) were in Stage II, 12 of them (14.12\%) with B symptoms, 31 patients (36.47\%) were in Stage III, 9 of them (10.59\%) with Stage III B and 8 of patients (9.41\%) were in Stage IV, three of them with Stage IV B.

According to $\mathrm{HL}$ risk categories that depend on the stage and number of nodal sites and the presence of bulky disease, favorable-risk pediatric Hodgkin lymphoma (stage IA or IIA with $<3$ nodal sites, and some IIIA without bulky disease) were occurred in 31 patients (36.47\%), intermediate-risk disease (stage IIA bulky disease with extension or $=3$ nodal sites, stage IB, IIB, stage IIIA, stage IVA) occurred in 42 patients (49.41\%) and advanced or unfavorable pediatric Hodgkin lymphoma that included all other patients that who were not included in the favorable or the intermediate risk groups) occurred in 12 patients (14.12\%).

This study showed that there is significant correlation between age and risk categories so that favorable risk category occurred more in younger age patients and high-risk category found in older children and adolescent (Table 2). This study found that there was statistic significant correlation ( $\mathrm{P}$-value $=0.03408)$ between age and sex as there was male predominance in young patients less than 10 years, while male to female ratio tend to be decrease with increasing age.

Table 3 shows age in relation to the sex ( $\mathrm{P}$-value $=0.03408$ ).

Also, there was significant correlation between Age and Histopathological subtypes of $\mathrm{HL}$, showing that MCHL found to be more common among children less than 10 years old, whereas NSHL found more in patients above 10 years old (P-Value $=0.05)$.

This study showed that there was significant statistic relation between histopathological 
subtypes and risk categories, showing that larger number of LDHL and NSHL fall in high risk group, whereas larger number of LRCHL and NLPHL fall in the favorable risk group (Table 4). There was no significant statistic relation between the site of the primary tumor and the risk categories in the current study $(\mathrm{P}-$ Value $=$ 0.78195).

Also, there was no significant statistic relation between risk categories and sex ( $\mathrm{P}$-Value = 0.66731).

Table 2. Relation between age and the risk categories

\begin{tabular}{cccc}
\hline Age in Years & \multicolumn{3}{c}{ Risk Categories Number/(Percentage) } \\
& $\begin{array}{c}\text { Favorable Risk } \\
\text { Group }\end{array}$ & $\begin{array}{c}\text { Intermediate Risk } \\
\text { Group }\end{array}$ & High Risk Group \\
\hline $0-4$ & $2(50.0 \%)$ & $1(25.0 \%)$ & $1(25.0 \%)$ \\
$5-9$ & $12(70.59 \%)$ & $4(23.53 \%)$ & $1(5.88 \%)$ \\
$10-14$ & $9(36.0 \%)$ & $13(52.0 \%)$ & $3(12.0 \%)$ \\
$15-18$ & $8(20.51 \%)$ & $24(61.54 \%)$ & $7(17.95 \%)$ \\
\hline
\end{tabular}

Chi-square test P-value $=0.02997$

Table 3. Age in relation to the sex

\begin{tabular}{ccc}
\hline Age in years & $\begin{array}{c}\text { Male } \\
\text { No. / total (\%) }\end{array}$ & $\begin{array}{c}\text { Female } \\
\text { No. / total (\%) }\end{array}$ \\
\hline $0-4$ & $3 / 4(75.0 \%)$ & $1 / 4(25.0 \%)$ \\
$5-9$ & $15 / 17(88.24 \%)$ & $2 / 17(23.53 \%)$ \\
$10-14$ & $20 / 25(80.0 \%)$ & $5 / 25(20.0 \%)$ \\
$15-18$ & $21 / 39(53.85 \%)$ & $18 / 39(46.15 \%)$ \\
\hline
\end{tabular}

Chi-square test P-value $=0.03408$

Table 4. The relation of histological subtype and risk categories risk categories NSHL

\begin{tabular}{cccccc}
\hline Risk & NSHL & MCHL & LRCHL & LDHL & NLPHL \\
Categories & No./Total (\%) & No./Total (\%) & No./Total (\%) & No./Total (\%) & No./Total (\%) \\
\hline Favorable & $17 / 31$ & $2 / 31$ & $9 / 31$ & $0 / 27$ & $2 / 31$ \\
Risk Group & $(54.84 \%)$ & $(6.45 \%)$ & $(29.0 \%)$ & $(0.0 \%)$ & $(6.45 \%)$ \\
\hline Intermediate & $21 / 42$ & $15 / 42$ & $3 / 42$ & $0 / 42$ & $3 / 42$ \\
Risk Group & $(50.0 \%)$ & $(35.71 \%)$ & $(7.14 \%)$ & $(0.0 \%)$ & $(7.14 \%)$ \\
\hline High Risk & $6 / 12$ & $5 / 12$ & $0 / 12$ & $1 / 12$ & $0 / 12$ \\
Group & $(50.0 \%)$ & $(41.7 \%)$ & $(0.0 \%)$ & $(8.3 \%)$ & $(0.0 \%)$ \\
\hline
\end{tabular}

Chi-square test $\mathrm{P}$-value $=0.00827$

\section{Discussion}

Pediatric and adolescent HL cancer continues to be the leading cause of death in children younger than 15 years old, and lymphomas are among the most common cancers seen in children. Fortunately, survival rates for childhood cancers have increased significantly over the years. Children respond to and deal with chemotherapy better than adults. Today, $96 \%$ of children diagnosed with Hodgkin's disease will survive 5 or more years ${ }^{(7)}$. However; 
those with high-risk disease continue to have poor outcomes.

The total number of cases studied was eightyfive, over a period of eight years. It found that males were affected more than females with The male:female ratio of 2.27:1; also our study found that there was significant correlation between age and sex as there was more male predominance in young patients less than 10 years, while male to female ratio tend to be decrease with increasing age, this figure is similar to male: female ratio in other studies which showed that children younger than 5 years show a strong male predominance $(M: F=$ $5: 3)$ and children aged 15 to 19 years show a slight female predominance $(\mathrm{M}: \mathrm{F}=0.8)^{(3,7)}$.

Peak age incidence at presentation in present study was $15-18$ years which was represent $45.9 \%$ of cases, In the USA, the incidence of $\mathrm{HL}$ is age-related and is highest among adolescents aged 15 to 19 years ( 29 cases per million per year), whereas children ages 10 to 14 years, 5 to 9 years, and 0 to 4 years having approximately threefold, eightfold, and 30-fold lower rates, respectively. In non-European Union countries, there is a similar rate in young adults but a much higher incidence in childhood (7).

In the current study the median age at diagnosis was about 13.7 years which was lower than that showed by study done in USA (median age: 15.6) ${ }^{(8,9)}$.

This younger median age at diagnosis in the present study might be due to the high incidence of EB virus infection in earlier age in our region; however, this concept should be confirmed by studying the EB virus genomes in the HL cells.

This study had concluded that pediatric HL was higher in male than female in our community. A median age at diagnosis was about 13.7 years with peak age of incidence between $15-18$ years and the majority of the patients presented with cervical lymph nodes primary at the time of diagnosis. Nodular sclerosis histopathology is the most common.

Most of the patients had stage II with Intermediate risk category.

There was strong correlation between age and advanced stage which meant that stage is age dependent and significant correlation between age and sex as there was more male predominance in young patients less than 10 years. Also, there was significant correlation between age and histopathology of $\mathrm{HL}$ in our study with mixed cellularity found to be more common among children less than 10 years old. Neither there was relation between the site of the primary tumor and the risk categories nor relation between risk categories and sex.

Despite absence of PET scan for the majority of patients for proposed risk stratification system for purpose of risk categories, significant number of our patients got intermediate and high-risk category.

\section{Acknowledgments}

None.

\section{Author contribution}

Dr. Abdallah: conducted obtaining consent, data collection, statistical analysis, and writing the manuscript. Dr. Rashid and Dr. Tawfiq: data collection, editing, and finalizing the writing of the study.

\section{Conflict of interest}

Authors declare no conflict of interest.

\section{Funding}

There is no funding source for this research.

\section{References}

1. Arya LS, Dinand V. Current strategies in the treatment of childhood Hodgkins disease. Indian Pediatr. 2005; 42(11): 1115-28.

2. Wikipedia - Hodgkin's lymphoma. URL: https://en.wikipedia.org/wiki/Hodgkin\%27s lympho ma. Accessed 21-6-2008.

3. Percy $C L$, Smith MA, Linet $M$, et al. Lymphomas and Reticuloendothelial Neoplasms. In: SEER Pediatric Monograph. Cancer incidence and survival among children and adolescents: united states SEER Program 1975-1995. National Cancer Institute. 1999.

4. Staal SP, Ambinder R, Beschorner WE, et al. A survey of Epstein-Barr virus DNA in lymphoid tissue. Frequent detection in Hodgkin's disease. Am J Clin Pathol. 1989; 91(1): 1-5.

5. Hjalgrim H, Askling J, Rostgaard K, et al. Characteristics of Hodgkin's lymphoma after infectious mononucleosis. N Engl J Med. 2003; 349(14): 1324-32. doi: 10.1056/NEJMoa023141.

6. Weiss LM, Chen YY, Liu XF, et al. Epstein-Barr virus and Hodgkin's disease. A correlative in situ hybridization 
and polymerase chain reaction study. Am J Pathol. 1991; 139(6): 1259-65.

7. Ries LA, Kosary CL, Hankey BF, et al., eds.. SEER Cancer Statistics Review 1973-1995. Bethesda, Md: National Cancer Institute, 1998.

8. Macfarlane GJ, Evstifeeva $T$, Boyle $P$, et al. International patterns in the occurrence of Hodgkin's disease in children and young adult males. Int J Cancer. 1995; 61(2): 165-9.

9. Best $T$, Li D, Andrew D Skol AD, et al. Variants at $6 q 21$ implicate PRDM1 in the etiology of therapy-induced second malignancies after Hodgkin's lymphoma Nat Med. 2011; 17(8): 941-3. doi: 10.1038/nm.2407.

Correspondence to Dr. Basil K. Abdallah E-mail: basilonc@yahoo.com Received Jan. $23^{\text {rd }} 2018$

Accepted Feb. 27 2019 